\title{
Clinical Significance of Caseating Granulomas Detected on Transrectal Ultrasound Biopsy of Prostate Gland: The Recommended Management
}

\author{
Salauddin SA ${ }^{\mathrm{a}}$, Sathiyananthan $\mathrm{JR}^{\mathrm{a}}$, Malek $\mathrm{R}^{\mathrm{a}}$ \\ ${ }^{a}$ Department of Urology, Selayang Hospital, Lebuhraya Selayang-Kepong Selangor, Malaysia
}

Keywords

Caseating, Granuloma, Acid fast bacilli,

Tuberculosis, Prostatitis

Corresponding Author

Syahril Anuar Salauddin

Department of Urology, Selayang Hospital,

68100 Batu Caves, Selangor, Malaysia.

Tel No: +60 162925506

E-mail: profunderground@yahoo.co.uk

Received: 24 August 2019, Accepted: 5 April 2021

Doi: https://doi.org/10.31436/imjm.v20i3

\begin{abstract}
Introduction: The histopathological examination (HPE) findings of caseating granulomas from the transrectal ultrasound (TRUS) of prostate biopsy are rare. A high index of suspicion is necessary to diagnose a tuberculosis infection. Here, we present the clinical outcomes of patients and the practical management steps when encounter these findings. Methods: We conducted a retrospective analysis of three cases in a tertiary care centre and reviewed the available literature on the topic. Results: All patients were aged less than 55 years and were considered young adults. One patient presented with neurogenic systemic symptoms, and two patients presented with lower urinary tract symptoms. All patients had abnormal digital rectal examination findings that warranted a TRUS biopsy of the prostate. All biopsies reported caseating granuloma with multinucleated giant cells. Additional investigations led to the diagnosis of tuberculosis infections in two patients, and one patient was diagnosed as having non-specific granulomatous prostatitis. All patients were treated according to their specific diagnosis and recovered fully. Conclusion: The detection of caseating granuloma on prostate biopsy may lead to diagnoses of several conditions, either infective or non-infective in origin. Further investigation is

mandatory to provide appropriate treatment.
\end{abstract}

\section{INTRODUCTION}

Tuberculosis (TB) of the prostate is a rare condition and is usually detected incidentally on histopathology examination (HPE). Due to the rarity of the condition, many urologists are unaware of this condition. Histopathological findings of a caseating granuloma from a transrectal ultrasound (TRUS) biopsy sample of the prostate should alert urologists to investigate further for TB prostatitis. Here, we present our steps of investigation based on three cases, wherein the HPE of the TRUS biopsy samples revealed a caseating granuloma, which, in turn, led to the diagnosis and appropriate management of different conditions.

\section{CASE 1}

A 44-year-old man presented with painless left testicular swelling, intermittent seizures, and significant weight loss for a duration of 4 months. Imaging showed multiple small nodular lesions in his brain parenchyma, pleura, and prostate. A digital rectal examination (DRE) revealed a hard and fixed prostate with multiple nodules. The initial tumour marker and test for TB were all negative. He was then referred to us for TRUS biopsy based on suspicion of advanced malignancy. HPE showed a benign prostatic gland with a focal area of necrosis, epithelioid granuloma, and occasional multinucleated giant cell that concluded as 
caseating granuloma with multinucleated giant cell. Additional staining using the Ziehl-Neelsen stain revealed acid-fast bacilli (AFB; Figure 1). He was then diagnosed with disseminated TB and treated with antiTB regime. Despite completing regular regime of 2 months induction and 6 months maintenance, repeated MRI brain showed persistent active lesion. Hence, additional 8 months maintenance regime was given. Clinically, he recovered, and the repeated scan showed resolution of all the aforementioned findings.

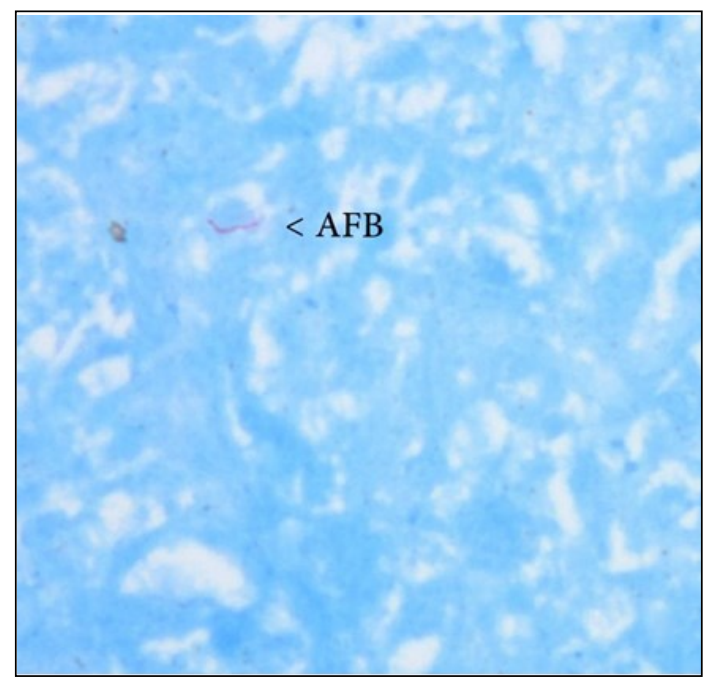

Figure 1: Histopathology slide shows acid fast bacilli (AFB) smear on Ziehl-Neelsen stain

\section{CASE 2}

A 54-year-old man presented with symptoms of urinary tract infection and acute urinary retention. A huge nodular prostate was palpated on DRE. A urinary catheter was inserted, and a course of antibiotics was prescribed. He was also administered an alpha-blocker as treatment for benign prostatic hyperplasia (BPH). Upon removal of the catheter, he was able to pass urine well and was referred to us for a TRUS biopsy due to abnormal DRE findings. The initial impression was prostate malignancy although his serum prostatic serum antigen (PSA) level was only $2.23 \mathrm{ng} / \mathrm{dL}$. The HPE findings of the TRUS biopsy sample revealed a caseating granuloma (Figure 2). A series of investigations were performed, including chest radiography, Mantoux test, assessment of serum erythrocyte sedimentation rate (ESR), sputum smear for $\mathrm{AFB}$, urine smear for $\mathrm{AFB}$, urine culture for $\mathrm{AFB}$, and urine TB analysis using polymerase chain reaction (PCR). Positive results were only obtained for the urine PCR. He was then diagnosed with TB prostatitis and treated with anti-TB regime for a duration of 9 months. Currently, his urination is normal, and serial uroflowmetry shows good urine flow.

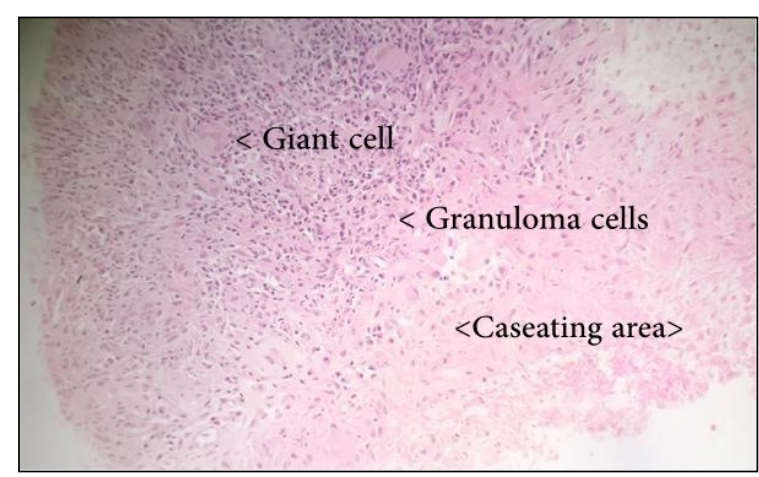

Figure 2. Histopathology slide of caseating granuloma from TRUS biopsy of prostate

\section{CASE 3}

A 52-year-old man was referred to us for further management of multiple thin anterior urethral strictures. He presented with lower urinary tract symptoms with recurrent urinary retention. He was initially treated for urinary tract infection with multiple courses of antibiotics. Further workup revealed a suspicious DRE finding of a hard nodule in his right lobe and a high PSA level of $7.38 \mathrm{ng} / \mathrm{L}$. He then underwent a TRUS biopsy and urethral dilatation with subsequent clean intermittent self-catherization. The HPE revealed a caseating granuloma with multiple multinucleated giant cells; no prostate malignancy was detected. All series of investigations for TB infection were negative. On follow-up, his PSA levels were below $4 \mathrm{ng} / \mathrm{L}$. He was diagnosed with non-specific granulomatous prostatitis. For 6 months, he performed regular continuous intermittent self-catheterization (CISC) and was able to pass urine without any difficulty. Presently, five years after the procedure, no recurrent urethral strictures have been noted.

\section{DISCUSSION}

According to the 2018 WHO Global Tuberculosis Report, the highest incidence of TB was in South-East Asia, accounting for $45 \%$ of the worldwide incidence of TB. ${ }^{1}$ Due to the rarity of non-pulmonary TB, such as genitourinary $\mathrm{TB}$, it is more difficult to diagnose. A caseating granuloma detected on TRUS biopsy may be the first finding indicting the diagnosis of a nonpulmonary TB infection. 
The incidence of caseating granulomas detected on TRUS biopsy was low, ranging from $0.36 \%$ to $1.86 \%{ }^{2,3}$ In 1984, Epstein and Hutchin classified the aetiology for caseating granulomas as follows: nonspecific (77.7\%), infective, such as TB infection (18.1\%), and others $(4.2 \%)$, such as iatrogenic (post prostatic surgery or radiation therapy) or cases associated with systemic granulomatous disease (sarcoidosis, rheumatoid arthritis, Wegener's granulomatosis, polyarteritis nodosa, and Churg-Strauss syndrome).3,4

The patients with TB prostatitis may present either with primary pulmonary symptoms followed by urinary tract symptoms or primary urinary tract symptoms and their complications. The patient in Case 1 presented with symptoms of TB in other organs and the patient in Case 2 presented with only urinary tract symptoms. In our series, there was a trend of caseating granuloma findings from HPE detected in patients who presented with symptoms and signs mimicking prostate cancer. In the other spectrum, many literatures reported patients who presented with complications of urinary $\mathrm{TB}$, such as multiple ureteric strictures, bladder contracture, or recurrent urethral stenosis. The intravenous urography finding of a corkscrew appearance and cystogram finding of a thimble bladder are examples of complications due to genitourinary tract TB infection. At this point, the management is challenging and usually requires repeated surgical interventions.

In our hospital, the detection of a caseating granuloma in a TRUS biopsy sample warrants several investigations, starting with the Mantoux test, chest radiography, serum ESR analysis, sputum AFB smear, urine AFB smear, urine culture for $\mathrm{AFB}$, and urine PCR. From the same biopsy slide, a pathologist can identify the AFB bacteria using the Ziehl-Neelsen stain. Despite the high specificity, the Ziehl-Neelsen stain has a low sensitivity of only $52.7 \%$. An 8 -week period is required to obtain an $\mathrm{AFB}$ urine culture with maximal sensitivity. The sensitivity and specificity of urine PCR for detection of a TB infection are high, being 95.59\% and $98.12 \%$, respectively. ${ }^{5}$ If the investigation is performed by an expert, the result can be obtained within one day. All these investigations are important to diagnose TB infection and determine the subsequent direction of management.
Definitive treatment depends on the patient's presentation and underlying aetiology. Non-specific granulomatous prostatitis usually resolves naturally, while infective granulomatous prostatitis requires medical treatment. The current standard treatment for infective TB prostatitis involves administration of 4 antibiotics during induction period for 2 months and 2 antibiotics for 6 months during maintenance period. ${ }^{6}$ The anti-TB regime is a multidrug treatment that is now available as a single tablet to allow for maximal patient tolerance and compliance. Some immunocompromised patients or patients with extensive infections may require treatment for a longer duration depending on the imaging and clinical responses, as seen in Case 1. Specifically, for TB prostatitis, additional quinolone group of antibiotics has showed better response compare to traditional regime. ${ }^{6}$ Treatment response is the best monitor through clinical observation in standardised clinical follow-ups. Surgery plays an important role as a second line treatment in cases of resistance to medical therapy or as part of a life-saving strategy. After completion of treatment, it is also recommended that patients should continue the follow-up, as the disease may relapse.

\section{CONCLUSION}

A caseating granuloma is rarely detected on TRUS biopsy. A high index of suspicion for TB is necessary to achieve the appropriate diagnosis. Investigations, including bedside tests, blood and urine analysis, and imaging are important to aid in the subsequent treatment. Management of the disease is based on the individual presentation and aetiology using medical or surgical therapy.

\section{ACKNOWLEDGEMENT}

We would like to show our gratitude to all our colleagues in Department of Urology, Selayang Hospital who provided expertise to produce this manuscript. We also thank to our pathologist from Department of Pathology, Selayang Hospital for sharing their thought with us. 


\section{REFERENCES}

1. Anderson L, Baddeley A, Dias HM et al, Global Tuberculosis Report 2018, World Health Organization, 26 Sep 2018.

2. Oppenheimer JR, Kahane H, Epstein JI. Granulomatous prostatitis on needle biopsy. Arch Pathol Lab Med. 1997 Jul;121(7):724-9.

3. Shukla P, Gulwani HV, Kaur S. Granulomatous prostatitis: clinical and histomorphologic survey of the disease in a tertiary care hospital. Prostate Int. 2017 Mar;5(1):29-34.

4. Kumbar R, Dravid N, Nikumbh D, et al.

Clinicopathological overview of granulomatous prostatitis: an appraisal. J Clin Diagn Res. 2016 Jan;10(1):EC20-3.

5. Singh J, Sharma P, Vijay MK, et al. Tuberculosis of the prostate: four cases and a review of the literature. UroToday Int J. 2013 Feb;6(1):1-3.

6. Kulchavenya E, Brizhatyuk E, Khomyakov V. Diagnosis and therapy for prostate tuberculosis. Ther Adv Urol 2014:6(4);129-34. 